\title{
Understanding hidden perspectives in biology of cancer - approach to its prevention and management
}

\begin{abstract}
Recent tumor genome sequencing confirmed that one tumor often consists of multiple cell subpopulations (clones) which bear different, but related, genetic profiles such as mutation and copy number variation profiles. Thus far, one tumor has been viewed as a whole entity in cancer functional studies. The adaptive immune response is controlled by checkpoints represented by coinhibitory molecules, which are crucial for maintaining self-tolerance and minimizing collateral tissue damage under physiological conditions. A growing body of preclinical evidence supports the hypothesis that unleashing this immunological break might be therapeutically beneficial in the fight against cancer, as it would elicit an effective antitumor immune response. Autophagy, which degrades redundant or damaged cellular constituents, is intricately relevant to a variety of human diseases, most notably cancer. Autophagy exerts distinct effects on cancer initiation and progression, due to the intrinsic overlapping of autophagic and cancer signalling pathways. Challenges to successfully treat cancer cells include the fact that they utilize endogenous mechanisms of cell growth and division, and that they are genetically unique to each patient and to each form of cancer. In order to identify successful pharmacotherapies to selectively target and treat cancer cells, an improved understanding of cellular processes involving oncogenesis as well as cancer cell growth and metastasis must be obtained. Together, these hidden facts may not only improve our understanding on cancer and its relationships, but also facilitate cancer drug discovery.
\end{abstract}

\author{
Volume 3 Issue 5 - 2015
}

\author{
Jatinder Pal Singh Chawla,' Gurmukh \\ Dhaliwal,' Inderjit Dhaliwal' \\ 'Department of oral and maxillofacial surgery, MM College of \\ Dental Sciences \& Research, India \\ ${ }^{2}$ Department of Dental Hygiene, Greenplaza Dental Centre, \\ USA
}

\section{Correspondence: Jatinder Pal Singh Chawla, Post Graduate} student, Department of oral and maxillofacial surgery, MM College of Dental Sciences \& Research, MMU, Mullana,Ambala, India,Tel 9729323332, Email jatinder.jps@rediffmail.com

Received: February 16, 2015 | Published: June 02, 2015

Keywords: cancer, biology, autophagy, mirna, cancer therapy, prevention

\section{Introduction}

Cancer is a disease mainly derived from mutations in single somatic cells that deviate from the normal routes of proliferation, migrate to adjacent normal tissues, and end in secondary tumors (metastasis) on sites different from the initial origin. In human beings, cancer refers to at least 100 versions of a disease that can develop in almost any tissue in the body. Despite the fact that each cancer type has unique attributes, all these different tumors evolve according to a common scheme of progression that includes genetic and epigenetic incidents in addition to a complex network of inter-actions between cells and the extracellular matrix in the host tissue. Cancerous cells communicate with their micro-environment in a way that can promote their growth, survival and the manifestation of distant metastasis. ${ }^{1}$ Changes in the genomes of cancer cells are the major cause of cancer. The power and efforts of sequence present in the tumour genome allow comprehensive cataloguing of genomic alterations. If collected, these catalogs have already shown that the main alterations in cancer are present in the key mutated genes and pathways. EGFR, RAS, PI3K, P53, FGFR, MET and many other well-known cancer-driver mutating genes have been frequently rediscovered in many tumor genome sequencing studies..$^{2-4}$ These facts strongly confirmed that the major cancer biology collections are generated via previous evident studies. On the other side, the cataloguing activity sometimes not able to provide many novel insights into the fundamental cancer biology, and the catalogs alone may not unveil new cancer treatment strategies for cancer management or cure. However, the tumor sequencing activity does provide multiple types of data for tumors: mutation, $\mathrm{CNVs}$, epigenetic and gene expression profiles.

\section{Coinhibitory molecules and immunosuppressive cell circuits}

The two-signal model states that the pivotal role at immunological synapse between a T-cell and an antigen presenting cell (APC) is dominantly played by cosignaling molecules. Specifically, optimal T-cell activation requires both the interaction between the T-cell receptor (TCR) and an antigen-derived peptide presented by major Histocompatibility complex (MHC) molecules (first signal), and the engagement of costimulatory molecules expressed on the surface of both the T-cell (i.e. CD28) and the APC (i.e. B7-1, B72) (second signal) $)^{5}$ : the failure of the T-cell to receive the second signal is associated to immunological anergy. ${ }^{6}$ On the other hand, coinhibitory molecules always prevent immune over activation and host tissue damage by limiting T-cell activity. ${ }^{7}$ Overall, cosignaling molecules cooperate to modulate the TCR signaling and ultimately to regulate T-cell mediated immunity towards dangerous entities such as infectious agents and malignant cells. Cosignaling molecules, which is either the tumor necrosis factor (TNF) or the immunoglobulin ligand or receptor super family, can be categorized into co-stimulatory and co-inhibitory molecules based on their respectively positive or negative effect on TCR-mediated immune responses. However, a typical feature of immune cosignaling ligands is that they can play a stimulatory or inhibitory role depending on what receptor they interact with, as exemplified by the opposite effects of B7-1 and B7-2 when they bind to CD28 or cytotoxic T lymphocyte associated antigen-4 (CTLA4), respectively. ${ }^{7}$

To make things more complicated, a co-signaling ligand can also act as a receptor (a phenomenon called reverse signaling), as it occurs 
when Treg cells (effector) inhibit the activity of DCs (target) through the interaction between the formal receptor CTLA4 (expressed on effector cells) and the formal ligands B7-1 and B7-2 (expressed on target cells). ${ }^{7}$ Finally, a ligand can even function as a conter-receptor for another ligand, as it is the case of B7-1 and programmed cell death ligand-1 (PDL1) in the inhibition of T-cell responses. ${ }^{8}$ The coexistence of cosignaling molecules with opposing functions corresponds to the need of controlling the offensive power of the individual's immune system under different physiological and pathological conditions (immune homeostasis), the ultimate goal being to achieve the elimination or control of (typically) a given pathogen with the minimum damage for the healthy tissues.

\section{Autophagy vs cancer therapy}

Autophagy (referring to macroautophagy) is an evolutionarily conserved cellular mechanism for the clearance of damaged or superfluous macro-complexes and organelles in eukaryotic cells. As the term auto (self)-phagy (eat) indicates, autophagy is a form of selfeating, which functions significantly in, for instance, housekeeping, cellular differentiation, growth control, cell defence, tissue remodelling, and acclimatisation. ${ }^{9}$ In autophagy, the double- or multimembrane-delimited autophagosome sequesters the cytoplasm in a non-specific way, and later fuses with the lysosome for degradation. ${ }^{10}$ Autophagy is strictly regulated by a limited number of autophagyrelated genes (ATGs) which were originally discovered in yeast, and over 20 of them have been identified in mammalian cells. ${ }^{11}$ ATGs play significant roles in autophagosome formation and autophagy regulation. Moreover, they have numerous links with many human diseases, particularly cancers. ${ }^{12}$ In cancer cells, autophagy promotes cell survival during metabolic stress, while also serving as a trigger of cell death. Therefore, autophagy seems contradictory with roles that support both pro-survival and pro-death pathways. ${ }^{13}$

Because it is a dynamic process, autophagy can be difficult to measure. Currently, the molecular mechanisms of autophagy and the role of autophagy in cancer cells are not clearly defined, but they are beginning to be revealed. Moreover, the role of autophagy varies depending on the particular type of cancer and the stage of disease progression. ${ }^{14}$ Generally, autophagy acts as a tumour suppressor at the early stages of tumour development. Expression of Beclin-1, a haploinsufficient tumour suppressor, reduces the oncogenic capacity through the induction of autophagy. Development, however, autophagy promotes tumour progression. For instance, autophagy helps cancer cells located in the central area of the tumour mass to survive lowoxygen and low-nutrient conditions. ${ }^{15-17}$ Another hypothesis suggests that autophagy can regulate tumourigenesis in a cell- or tissue-specific manner. ${ }^{18,19}$ Autophagy is a necessary and effective method employed by tumours to survive a dormancy period..$^{20}$ Furthermore, autophagy can impact the regulation of cell growth. In apoptosis- defective metabolically stressed tumour cells, the initiation of autophagy prevents cell death from necrosis, avoiding the exacerbation of local inflammation and subsequent aggressive tumour growth. Seen from the long term, however, autophagy limits the blood supply of tumour cells and impedes further tumour development. ${ }^{21}$ As discussed above, due to the complicated roles of autophagy in cancer cells, inducing or inhibiting autophagy as the therapeutic method for cancer depends on various factors including the type of cancer, the progression of the disease and the nature of the treatment.

\section{MicroRNAs in cancer biology and therapy}

The discovery of lin-4 in Caenorhabditis elegans, the first microRNA (miRNA), led to the identification of several hundred other
miRNA molecules..$^{22,23}$ MiRNAs are short noncoding RNA molecules of roughly 19-24 nucleotides in length and are a large class, with more than 1,000 members, of small-regulatory RNAs in mammalian genomes. A single miRNA may play distinct roles in different tissues. For instance, the miR-181 family has traditionally been described in several solid malignancies, such as breast, liver and colon cancer, as an oncomiR (defined as a miRNA that acts as an oncogene to promote tumorigenesis and tumor progression); however, in AML this family acts as tumor suppressor. Due to the central role of miRNAs in cancer initiation and progression, they have been a source of interest for several years, specifically whether these miRNAs can be targeted or not. ${ }^{24}$ As already described, it has been observed that several drugs can alter miRNA expression. Moreover, if certain cancers are particularly "addicted" to particular miRNAs (named oncomiR addiction) then targeting specific miRNAs selectively should minimize off-target toxicity. ${ }^{25}$ Investigators are interested in designing inhibitors for oncogenic miRNAs and mimics for tumor-suppressor miRNAs that can act alone or synergistically with currently approved treatments. Currently, there are dozens of clinical trials that are assessing the correlation between miRNA expression and cancer diagnosis and prognosis. Several of these trials have been started in the past five years (www.clinicaltrials.gov) while new ones are being designed. Due to the pleiotropic effects of miRNAs they have been an attractive avenue for patient diagnosis evaluation and prognosis. Also, miRNAs, attributed to their size, are highly stable and resistant to RNAses and thus have a higher level of stability than mRNA. The future work in the next ten years will be focused on understanding how miRNAs regulate target genes in cancer initiation, progression, metastasis, relapse, and drug response and resistance, which will provide a more comprehensive mechanistic analysis of these noncoding RNAs. Furthermore, more noncoding RNAs are constantly being studied and are also an increasing area of focus. Future work will undoubtedly focus on the co-interaction and regulation of noncoding RNAs and how they together contribute to disease. Once we have gained this understanding, it will allow researchers to design better miRNA inhibitors or miRNA mimics that will guide the creation of new and more effective drug therapies.

\section{Further advances in biology of cancer}

The incidence of oral cancer remains high and is associated with many deaths in both Western and Asian countries. Several risk factors for the development of oral cancer are now well known, including smoking, drinking and consumption of smokeless tobacco products. Genetic predisposition to oral cancer has been found in certain cases but its components are not yet entirely clear. In accordance with the multi-step theory of carcinogenesis, the natural history of oral cancer seems to gradually evolve through transitional precursor lesions from normal epithelium to a full-blown metastatic phenotype. A number of genomic lesions accompany this transformation and a wealth of related results has appeared in recent literature and is being summarized here. Furthermore, several key genes have been implicated, especially wellknown tumor suppressors like the cyclin-dependent kinase inhibitors, TP53 and RB1 and oncogenes like the cyclin family, EGFR and ras. Viral infections, particularly with oncogenic HPV subtypes and $\mathrm{EBV}$, can have a tumorigenic effect on oral epithelia and their role is discussed, along with potential therapeutic interventions.

\section{Conclusion}

The study of oral cancer is particularly challenging. Oral cancer is an important cause of morbidity and mortality, especially in developing countries, and its prevalence may rise in the foreseeable 
future. Advances in diagnosis and treatment have slowly accumulated, but a sound understanding of underlying cell biology is likely to enable further, much-needed progress.

\section{Acknowledgements}

None.

\section{Conflicts of interest}

The authors declare that there is no conflict of interest.

\section{References}

1. Martins ML, Ferreira SC, Vilela MJ. Multiscale models for the growth of avascular tumors. Phys Life Rev. 2007;4(2):128-125.

2. Network CGA. Comprehensive molecular portraits of human breast tumours. Nature. 2012;490(7418):61-70.

3. Network CGAR. Comprehensive genomic characterization of squamous cell lung cancers. Nature. 2012;489(7417):519-525.

4. Network CGAR. Integrated genomic analyses of ovarian carcinoma. Nature. 2011;474(7353):609-615.

5. Carreno BM, Collins M. The B7 family of ligands and its receptors: new pathways for costimulation and inhibition of immune responses. Annual Review of Immunology. 2002;20:29-53.

6. Schwartz RH. T cell anergy. Annu Rev Immunol. 2003;21:305-334.

7. Chen L. Co-inhibitory molecules of the B7-CD28 family in the control of T-cell immunity. Nature Rev Immunol. 2004;4(5):336-347.

8. Butte MJ, Keir ME, Phamduy TB, et al. Programmed death-1 ligand 1 interacts specifically with the B7-1 costimulatory molecule to inhibit $\mathrm{T}$ cell responses. Immunity. 2007;27(1):111-122.

9. Klionsky DJ, Emr SD. Autophagy as a regulated pathway of cellular degradation. Science. 2000;290(5497):1717-1721.

10. Mizushima N, Klionsky DJ. Protein turnover via autophagy: implications for metabolism. Annu Rev Nutr. 2007;27:19-40.

11. Mizushima N, Levine B, Cuervo AM, et al. Autophagy fights disease through cellular self-digestion. Nature. 2008;451(7182):1069-1075.
12. Kung CP, Budina A, Balaburski G, et al. Autophagy in tumor suppression and cancer therapy. Crit Rev Eukaryot Gene Expr. 2011;21(1):71-100.

13. Baehrecke EH. Autophagy: dual roles in life and death?. Nat Rev Mol Cell Biol. 2005;6(6):505-510.

14. Hippert MM, O’Toole PS, Thorburn A. Autophagy in cancer: good, bad, or both?. Cancer Res. 2006;66(19):9349-9351.

15. Cuervo AM. Autophagy: in sickness and in health. Trends Cell Biol. 2004;14(2):70-77.

16. Kondo Y, Kanzawa T, Sawaya R, et al. The role of autophagy in cancer development and response to therapy. Nat Rev Cancer. 2005;5(9):726734 .

17. Liang $\mathrm{C}$, Feng $\mathrm{P}, \mathrm{Ku} \mathrm{B}$, et al. Autophagic and tumour suppressor activity of a novel Beclin1-binding protein UVRAG. Nat Cell Biol. 2006;8(7):688-699.

18. Mathew R, Karantza-Wadsworth V, White E. Role of autophagy in cancer. Nat Rev Cancer. 2007;7:961-967.

19. Gozuacik D, Kimchi A. Autophagy as a cell death and tumor suppressor mechanism. Oncogene. 2004;23(16):2891-2906.

20. Lu Z, Luo RZ, Lu Y, et al. The tumor suppressor gene ARHI regulates autophagy and tumor dormancy in human ovarian cancer cells. J Clin Invest. 2008;118:3917-3929.

21. Eisenberg-Lerner A, Bialik S, Simon HU, et al. Life and death partners: apoptosis, autophagy and the cross-talk between them. Cell Death Differ. 2009;16(7):966-975.

22. Lee RC, Feinbaum RL, Ambros V. The C Elegans heterochronic gene lin-4 encodes small RNAs with antisense complementarity to lin-14. Cell. 1993;75(5):843-854.

23. Wightman B, Ha I, Ruvkun G. Posttranscriptional regulation of the heterochronic gene lin-14 by lin-4 mediates temporal pattern formation in C. elegans. Cell. 1993;75(5):855-862.

24. Watashi K, Yeung ML, Starost MF, et al. Identification of small molecules that suppress microRNA function and reverse tumorigenesis. J Biol Chem. 2010;285(32):24707-24716.

25. Cheng CJ, Slack FJ. The duality of oncomiR addiction in the maintenance and treatment of cancer. Cancer J. 2012;18(3):232-237. 